\section{CBT improves maternal perinatal depression in rural Pakistan}

\section{QUESTION}

Question: Does a community health worker delivered cognitive behavioural therapy (CBT) based intervention improve the health of perinatally depressed women and their newborn infants in rural Pakistan?

Patients: 903 married women, aged 16-45 years, in their third trimester of pregnancy and diagnosed with DSM-IV perinatal major depression (CBT based intervention, 463; control, 440). Exclusions for mothers: serious medical condition; pregnancy related illness; disability; psychosis; and serious illness, death or moved residence during the study. Exclusions for infants: born prematurely or with congenital defect; aborted or stillborn; died within first year of birth; and given up for adoption.

Setting: 40 Union Councils (administrative units) in Gujar Khan and Kallar Syedan, rural Pakistan. Recruitment: April 2005 to March 2006.

Intervention: $\mathrm{CBT}$ based intervention or enhanced routine care (control). Both groups received visits from local female health visitors every week during the last month of pregnancy, three times in the month after birth and then once a month for 9 months. The intervention group health workers were trained to deliver the CBT based Thinking Healthy Programme using a manual with stepwise instructions. The programme involves active listening, collaboration with the family, probing health beliefs and generating alternate ways of thinking.

Outcomes: Primary outcomes: infant weight and height ( $\mathrm{z}$ scores, ie, numbers of standard deviations away from the mean). Secondary outcomes: maternal depression (DSM-IV diagnoses; Hamilton Depression Rating Scale), disability (Brief Disability Questionnaire), functioning (Global Assessment of Functioning Scale) and perceived social support (multidimensional 12 item Likert scale); complete immunisation of infant; and use of any form of contraception.

Patient follow-up: $88 \%$ of mothers; $78 \%$ of infants $(10 \%$ of infants did not meet the inclusion criteria).

\section{METHODS}

Design: Clustered randomised controlled trial (Union Councils were the unit of randomisation).

Allocation: Concealed.

Blinding: Single blind (assessors blinded).

Follow-up period: 12 months (after childbirth).

\section{MAIN RESULTS:}

At 12 months after childbirth, there was no difference in weight for age or height for age between infants from the CBT based intervention and control groups (adjusted mean difference height for age $\mathrm{z}$ score: $+0.17,95 \%$ CI -0.02 to +0.35 ; adjusted mean difference weight for age $\mathrm{z}$ score: +0.07 , $95 \%$ CI -0.08 to +0.22$)$. However, the CBT based intervention reduced the prevalence of DSM-IV major depression among mothers compared with the control $(27 \%$ with intervention vs $59 \%$ with control; adjusted odds ratio 0.23 , $95 \%$ CI 0.15 to 0.36 ). Compared with control, CBT reduced levels of depression symptoms and disability, and improved overall functioning and perceived social support. CBT also increased infant immunisation rates and uptake of contraception at 12 months (see online table).

\section{CONCLUSIONS}

The CBT based Thinking Healthy Programme delivered by health workers improves the mental health of perinatally depressed women in rural Pakistan. However, it does not improve indicators of infant growth at 1 year.

\section{ABSTRACTED FROM}

Rahman A, Malik A, Sikander S, et al. Cognitive behaviour therapy-based intervention by community health workers for mothers with depression and their infants in rural Pakistan: a cluster-randomised controlled trial. Lancet 2008;372:902-909.

Correspondence to: Professor Atif Rahman, University of Liverpool, School of Population, Community and Behavioural Sciences, Child Mental Health Unit, Alder Hey Children's NHS Foundation Trust, Mulberry House, Eaton Road, Liverpool, L12 2AP, UK; Atif.Rahman@liverpool.ac.uk

Source of funding: Wellcome Trust, UK.

- A reference list and a table are published online only at http://ebmh.bmj.com/ content/vol12/issue2
$\mathrm{R}$ ahman and colleagues add to a growing body of evidence ${ }^{1}$ regarding the effectiveness of organised depression care programmes for disadvantaged communities in low and middle income countries. Several rigorous trials in primary care and community settings now demonstrate that moderate investments in improved depression care yield even greater benefits in resource poor settings than in wealthy ones. Indigenous primary care or community health workers can be trained to provide effective psychological and behavioural therapies.

It is notable that depression care programmes developed for resource poor settings typically reverse the order of stepped care interventions developed in wealthier settings. The traditional stepped care approach begins with less expensive medication management in primary care, reserving more expensive psychotherapy or other specialty treatment for those with more severe or treatment resistant depression. When psychotherapy can be delivered by existing health workers, it becomes an economically viable firstline treatment for depression.

We should not be disappointed that this trial did not demonstrate significant effects of maternal depression treatment on infant growth and development. Their previous observational study ${ }^{2}$ found that children of depressed and non-depressed mothers differed in growth by approximately 0.6 standard deviations. The intervention reduced the prevalence of depression at follow-up by approximately $30 \%$. If we generously assume that the intervention completely eliminates all effects of depression in the additional 30\% who recover, we would still expect the intervention to yield, at most, a difference in growth rates of 0.18 standard deviations. Such a difference would certainly be important from a public health perspective but the current trial was not large enough to reliably detect it.

Of course, the primary goals of improving depression treatment are to relieve suffering and improve quality of life. Positive downstream effects of depression treatment (improved infant health, increased work productivity, decreased use of unnecessary medical services) are added bonuses.

\section{Gregory E Simon, MD, MPH}

Group Health Center for Health Studies, Seattle, Washington, USA

Competing interests: None. 